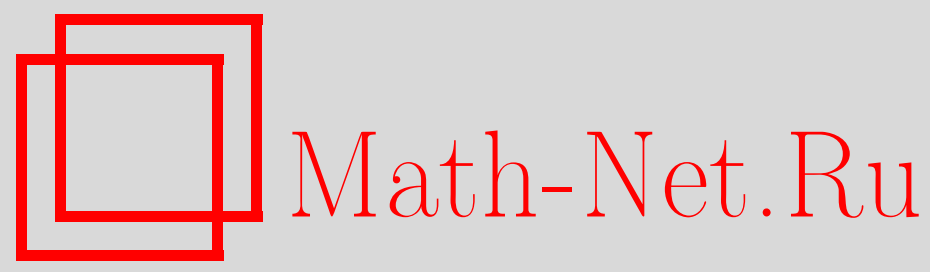

С. Д. Троицкая, О первой краевой задаче для гиперболического уравнения на плоскости, Матем. заметки, 1999, том 65, выпуск 2, 294-306

DOI: https://doi.org/10.4213/mzm1052

Использование Общероссийского математического портала Math-Net.Ru подразумевает, что вы прочитали и согласны с пользовательским соглашением http://www.mathnet.ru/rus/agreement

Параметры загрузки:

IP : 54.224 .135 .184

26 апреля 2023 г., 15:14:08 


\section{О ПЕРВОЙ КРАЕВОЙ ЗАДАЧЕ ДЛЯ ГИПЕРБОЛИЧЕСКОГО УРАВНЕНИЯ НА ПЛОСКОСТИ}

\section{С. Д. Троицкая}

В работе изучается краевая задача для гиперболического уравнения с двумя независимыми переменными, являющаяся обобщением известной задачи Дарбу.

Библиография: 14 названий.

Пусть $D_{*}$ - область в плоскости $O x y$, которая ограничена двумя гладкими кривыми $\Gamma_{1}:=\left\{(x, y): y=\gamma_{1}(x),-\infty<x_{*} \leqslant x \leqslant 0\right\}$ и $\Gamma_{2}:=\left\{(x, y): x=\gamma_{2}(y), 0 \leqslant y \leqslant y_{*}<\infty\right\}$, выходящими из начала координат и целиком лежащими в координатном угле $x \leqslant 0, y \geqslant 0$, а также отрезками прямых $x=x_{*}$ и $y=y_{*}$. В области $D_{*}$ рассмотрим гиперболическое уравнение

$$
\frac{\partial^{2} u}{\partial x \partial y}+a(x, y) \frac{\partial u}{\partial x}+b(x, y) \frac{\partial u}{\partial y}+c(x, y) u=f(x, y)
$$

в котором $a(x, y), b(x, y) \in C^{1}\left(D_{0}\right), c(x, y) \in C\left(D_{0}\right)$, где область $D_{0} \supset \bar{D}_{*}$, a $f \in L_{2}\left(D_{*}\right)$. Ниже мы будем рассматривать обобщенные решения $u(x, y)$ этого уравнения, принадлежащие пространству Соболева $W_{2}^{1}\left(D_{*}\right)$. Известно (см. [1]), что при выполнении определенных условий на расположение кривых $\Gamma_{i}$ (например, когда кривые $\Gamma_{i}$ совпадают с характеристиками уравнения (1), либо когда они ни в одной своей точке не имеют характеристического направления) всякое такое решение $u(x, y)$ почти всюду в $D_{*}$ совпадает с функцией $u_{c}(x, y)$, непрерывной на $\bar{D}_{*} \backslash D^{*}$, где множество $D^{*}$ состоит не более чем из двух точек. Более того, следы функции $u_{c}(x, y)$ на кривых $\Gamma_{1}, \Gamma_{2}$ принадлежат пространствам $W_{2}^{1}\left(x_{*}, \varepsilon\right), W_{2}^{1}\left(\varepsilon, y_{*}\right)$ соответственно для любого $\varepsilon>0$. Эти факты позволяют изучать краевую задачу, которая является естественньм обобщением задачи Дарбу и заключается в нахождении обобщенного решения $u(x, y)$ уравнения $(1)$, принадлежащего пространству $W_{2}^{1}\left(D_{*}\right)$ и удовлетворяющего краевым условиям вида

$$
\begin{gathered}
\left.u\right|_{\Gamma_{i}}=\varphi_{i}, \quad i=1,2, \quad \varphi_{1} \in W_{2}^{1}\left(x_{*}, 0\right), \quad \varphi_{2} \in W_{2}^{1}\left(0, y_{*}\right), \\
\varphi_{1}(0)=\varphi_{2}(0) .
\end{gathered}
$$

В большинстве работ изучаются частные случаи этой задачи, когда либо одна из кривых $\Gamma_{1}, \Gamma_{2}$, либо обе являются характеристиками, а $\varphi_{1}=\varphi_{2} \equiv 0$ (см. [2]-[6]).

Работа выполнена при частичной поддержке Российского фонда фундаментальных исследований, грант № 98-01-01000, и программы "Ведущие научные школы”, грант № 96-15-96091. 
Основной цель настоящей работы - изучение задачи (1), (2) в области с нехарактеристической границей. Именно такая задача возникает при исследовании спектральных свойств задачи Соболева о малых колебаниях вращающейся жидкости в некоторых трехмерных областях с ребрами (см. [1], [7]).

Обозначим через $C^{n, n}(S)$, где $S$ - некоторая область с кусочно-гладкой границей, множество всех функций $r(x, y)$, имеющих непрерьвные на $\bar{S}$ смешанные производные

$$
\frac{\partial^{i+j} r(x, y)}{\partial x^{i} \partial y^{j}}, \quad i, j=0,1, \ldots, n
$$

через $W_{2}^{n, n}(S), n \geqslant 0$, гильбертово пространство функций из $L_{2}(S)$ с нормой

$$
\|f\|_{W_{2}^{n, n}(S)}^{2}=\sum_{i, j=0}^{n} \iint_{S}\left|\frac{\partial^{i+j} f}{\partial x^{i} \partial y^{j}}\right|^{2} d x d y .
$$

Теорема 1. Пусть область $D:=\left\{(x, y): x_{*}<x<0,0<l_{2}(x)<y<l_{1}(x)\right\}$, әде функиии $l_{1}(x), l_{2}(x) \in C^{1}\left[x_{*}, 0\right]$ такие, что

$$
\begin{gathered}
l_{1}^{\prime}(x)<0, \quad l_{2}^{\prime}(x)<0 \quad \text { для всех } \quad x \in\left(x_{*}, 0\right), \\
l_{1}(0)=l_{2}(0)=0, \quad 0<\lim _{x \rightarrow-0}\left|l_{2}^{\prime}(x)\right|<\lim _{x \rightarrow-0}\left|l_{1}^{\prime}(x)\right| .
\end{gathered}
$$

Для любой функиии $f \in L_{2}(D)$, любых $\varphi_{1}, \varphi_{2} \in W_{2}^{1}\left(x_{*}, 0\right), \varphi_{1}(0)=\varphi_{2}(0)$, в пространстве $W_{2}^{1}(D)$ всегда существует и притом единственное обобщенное решение $u(x, y)$ уравнения $(1)$, удовлетворяющее условиям

$$
u\left(x, l_{i}(x)\right)=\varphi_{i}(x), \quad x \in\left(x_{*}, 0\right), \quad i=1,2,
$$

и для него справедлива оченка

$$
\|u\|_{W_{2}^{1,1}(D)}^{2} \leqslant c\left(\|f\|_{L_{2}(D)}^{2}+\left\|\varphi_{1}\right\|_{W_{2}^{1}\left(x_{*}, 0\right)}^{2}+\left\|\varphi_{2}\right\|_{W_{2}^{1}\left(x_{*}, 0\right)}^{2}\right)
$$

где с не зависит от $f, \varphi_{1}, \varphi_{2}$.

Если дополнительно предположить, что $a, b \in C^{n+1, n+1}\left(D_{0}\right), c \in C^{n, n}\left(D_{0}\right)$ $\left(D_{0} \supset \bar{D}\right), n \geqslant 0, l_{1}, l_{2} \in C^{n+1}\left[x_{*}, 0\right], f \in W_{2}^{n, n}(D)$, а функиии $\varphi_{1}, \varphi_{2} \in W_{2}^{n+1}\left(x_{*}, 0\right)$, то указанное решение $u(x, y)$ принадлежит пространству $W_{2}^{n+1, n+1}(D)$ и справедлива оченка

$$
\|u\|_{W_{2}^{n+1, n+1}(D)}^{2} \leqslant c_{n}\left(\|f\|_{W_{2}^{n, n}(D)}^{2}+\left\|\varphi_{1}\right\|_{W_{2}^{n+1}\left(x_{*}, 0\right)}^{2}+\left\|\varphi_{2}\right\|_{W_{2}^{n+1}\left(x_{*}, 0\right)}^{2}\right)
$$

әде $c_{n}$ не зависит от $f, \varphi_{1}, \varphi_{2}$.

Некоторые результаты настоящей работы анонсированы в [8].

В случае, когда $a=b=c \equiv 0$, a $\Gamma_{1}, \Gamma_{2}$ являются отрезками прямых, единственность обобщенного решения такой задачи в пространстве $W_{2}^{1}(D)$ фактически доказана в [7]. В [1] для произвольных кривых $\Gamma_{1}, \Gamma_{2}$, удовлетворяющих сформулированным условиям, единственность указанного решения доказана для уравнения Эйлера-Дарбу. 
1. О гладкости решений задачи Гурса. Для доказательства теоремы 1 нам понадобится следующее утверждение.

Лемма. Пусть в прямоугольной области $P=\left\{(x, y): x_{0}<x<x_{1}, y_{0}<y<y_{1}\right\}$ задано уравнение $(1)$, в котором $a(x, y), b(x, y), c(x, y), f(x, y) \in C^{n, n}(P), n \geqslant 0, u$ заданы функиии $\varphi_{1}(x) \in C^{n+1}\left[x_{0}, x_{1}\right], \varphi_{2}(y) \in C^{n+1}\left[y_{0}, y_{1}\right]$ maкие, что $\varphi_{1}\left(x_{0}\right)=\varphi_{2}\left(y_{0}\right)$. Тогда в классе $C^{1}(\bar{P})$ существует единственное решение $u(x, y)$ уравнения $(1)$, удовлетворяющее условиям

$$
u\left(x, y_{0}\right)=\varphi_{1}(x), \quad u\left(x_{0}, y\right)=\varphi_{2}(y)
$$

Это решение принадлежит классу $C^{n+1, n+1}(P)$.

ДокАЗАтЕЛЬСтво. Пусть $n=0$. Тогда, как известно, в классе $C^{1}(\bar{P})$ указанное решение $u(x, y)$ существует и единственно, при этом $\partial^{2} u / \partial x \partial y \in C(\bar{P})$, т.е. $u(x, y) \in$ $C^{1,1}(P)$. Докажем утверждение при $n=1$. Положим

$$
\begin{gathered}
t_{00}^{*}:=u, \quad t_{01}^{*}:=\frac{\partial u}{\partial y}, \quad t_{10}^{*}:=\frac{\partial u}{\partial x}, \quad t_{02}^{*}:=\frac{\partial^{2} u}{\partial y^{2}}, \quad t_{20}^{*}:=\frac{\partial^{2} u}{\partial x^{2}} \\
t_{11}^{*}:=\frac{\partial^{2} u}{\partial x \partial y}, \quad t_{12}^{*}:=\frac{\partial^{3} u}{\partial x \partial y^{2}}, \quad t_{21}^{*}:=\frac{\partial^{3} u}{\partial x^{2} \partial y}, \quad t_{22}^{*}:=\frac{\partial^{4} u}{\partial x^{2} \partial y^{2}}
\end{gathered}
$$

Дифференцируя систему интегральных уравнений (6) из [9, с. 253], к которой традиционно сводится задача Гурса, несложно показать, что $t_{i j}^{*}$ удовлетворяют следующей системе уравнений:

$$
\begin{gathered}
t_{00}^{*}=\varphi_{1}(x)+\int_{y_{0}}^{y} t_{01}^{*}\left(x, y^{\prime}\right) d y^{\prime}, \quad t_{10}^{*}=\varphi_{1}^{\prime}(x)+\int_{y_{0}}^{y}\left(F-a t_{10}^{*}-b t_{01}^{*}-c t_{00}^{*}\right)\left(x, y^{\prime}\right) d y^{\prime}, \\
t_{01}^{*}=\varphi_{2}^{\prime}(y)+\int_{x_{0}}^{x}\left(F-a t_{10}^{*}-b t_{01}^{*}-c t_{00}^{*}\right)\left(x^{\prime}, y\right) d x^{\prime}, \quad t_{11}^{*}=F-a t_{10}^{*}-b t_{01}^{*}-c t_{00}^{*}, \\
t_{20}^{*}=\varphi_{1}^{\prime \prime}(x)+\int_{y_{0}}^{y}\left(F_{x}^{\prime}-a t_{20}^{*}-b t_{11}^{*}-c t_{10}^{*}-a_{x}^{\prime} t_{10}^{*}-b_{x}^{\prime} t_{01}^{*}-c_{x}^{\prime} t_{00}^{*}\right)\left(x, y^{\prime}\right) d y^{\prime} \\
t_{02}^{*}=\varphi_{2}^{\prime \prime}(y)+\int_{x_{0}}^{x}\left(F_{y}^{\prime}-a t_{11}^{*}-b t_{02}^{*}-c t_{01}^{*}-a_{y}^{\prime} t_{10}^{*}-b_{y}^{\prime} t_{01}^{*}-c_{y}^{\prime} t_{00}^{*}\right)\left(x^{\prime}, y\right) d x^{\prime} \\
t_{21}^{*}=F_{x}^{\prime}-a t_{20}^{*}-b t_{11}^{*}-c t_{10}^{*}-a_{x}^{\prime} t_{10}^{*}-b_{x}^{\prime} t_{01}^{*}-c_{x}^{\prime} t_{00}^{*} \\
t_{12}^{*}=F_{y}^{\prime}-a t_{11}^{*}-b t_{02}^{*}-c t_{01}^{*}-a_{y}^{\prime} t_{10}^{*}-b_{y}^{\prime} t_{01}^{*}-c_{y}^{\prime} t_{00}^{*} \\
t_{22}^{*}=F_{x y}^{\prime \prime}-a t_{21}^{*}-b t_{12}^{*}-c t_{11}^{*}-a_{y}^{\prime} t_{20}^{*}-b_{y}^{\prime} t_{11}^{*}-c_{y}^{\prime} t_{10}^{*} \\
-a_{x}^{\prime} t_{11}^{*}-b_{x}^{\prime} t_{02}^{*}-c_{x}^{\prime} t_{01}^{*}-a_{x y}^{\prime \prime} t_{10}^{*}-b_{x y}^{\prime \prime} t_{01}^{*}-c_{x y}^{\prime \prime} t_{00}^{*} .
\end{gathered}
$$

Пусть $\{C(\bar{P})\}^{9}$ - пространство непрерывных на $\bar{P}$ вектор-функций

$$
\vec{t}^{*}=\left(t_{00}^{*}, t_{10}^{*}, t_{01}^{*}, t_{11}^{*}, t_{20}^{*}, t_{02}^{*}, t_{21}^{*}, t_{12}^{*}, t_{22}^{*}\right)
$$

с естественной нормой

$$
\left\|\vec{t}^{*}\right\|=\max _{i, j=0,1,2}\left(\max _{(x, y) \in \bar{P}}\left|t_{i j}^{*}(x, y)\right|\right) .
$$


Обозначим через $\mathscr{A}_{0}:\{C(\bar{P})\}^{9} \rightarrow\{C(\bar{P})\}^{9}$ линейньй оператор, определяемый соответствующей однородной системой уравнений, а через $\vec{\Phi} \in\{C(\bar{P})\}^{9}$ вектор с координатами

$$
\begin{gathered}
\Phi_{00}=\varphi_{1}(x), \quad \Phi_{10}=\varphi_{1}^{\prime}(x)+\int_{y_{0}}^{y} F\left(x, y^{\prime}\right) d y^{\prime}, \\
\Phi_{01}=\varphi_{2}^{\prime}(y)+\int_{x_{0}}^{x} F\left(x^{\prime}, y\right) d x^{\prime}, \quad \Phi_{11}=F, \\
\Phi_{20}=\varphi_{1}^{\prime \prime}(x)+\int_{y_{0}}^{y} F_{x}^{\prime}\left(x, y^{\prime}\right) d y^{\prime}, \quad \Phi_{02}=\varphi_{2}^{\prime \prime}(y)+\int_{x_{0}}^{x} F_{y}^{\prime}\left(x^{\prime}, y\right) d x^{\prime}, \\
\Phi_{21}=F_{x}^{\prime}, \quad \Phi_{12}=F_{y}^{\prime}, \quad \Phi_{22}=F_{x y}^{\prime \prime} .
\end{gathered}
$$

Рассмотрим отображение $\mathscr{A}\left(\vec{t}^{*}\right)=\vec{t}^{* *}, \mathscr{A}:\{C(\bar{P})\}^{9} \rightarrow\{C(\bar{P})\}^{9}$, определяемое формулой $\mathscr{A}\left(\vec{t}^{*}\right)=\vec{\Phi}+\mathscr{A}_{0}\left(\vec{t}^{* *}\right)$. Тогда, очевидно, решение нашей системы уравнений равносильно нахождению неподвижных точек отображения $\mathscr{A}$. Докажем, что некоторая степень отображения $\mathscr{A}$ является сжимаюшим отображением. Если $\mathscr{A}\left(\vec{s}^{*}\right)=\vec{\Phi}+\mathscr{A}_{0}\left(\vec{s}^{* *}\right)$, $\vec{s}^{*}, \vec{s}^{* *} \in\{C(\bar{P})\}^{9}$, то, очевидно, $\mathscr{A}^{k}\left(\vec{t}^{*}\right)-\mathscr{A}^{k}\left(\vec{s}^{*}\right)=\mathscr{A}_{0}^{k}\left(\vec{t}^{* *}-\vec{s}^{* *}\right), k=1,2, \ldots$ Положим

$$
\begin{gathered}
\vec{t}^{[0]}:=\vec{t}^{* *}-\vec{s}^{* *}, \quad \vec{t}^{[k]}:=\mathscr{A}_{0}^{k}\left(\vec{t}^{[0]}\right), \quad k=1,2, \ldots, \quad M:=\left\|\vec{t}^{[0]}\right\|, \\
K:=1+\max \left(|a|+|b|+|c|+\left|a_{x}^{\prime}\right|+\left|b_{x}^{\prime}\right|+\left|c_{x}^{\prime}\right|+\left|a_{y}^{\prime}\right|+\left|b_{y}^{\prime}\right|+\left|c_{y}^{\prime}\right|+\left|a_{x y}^{\prime \prime}\right|+\left|b_{x y}^{\prime \prime}\right|+\left|c_{x y}^{\prime \prime}\right|\right) .
\end{gathered}
$$

Тогда при $k \geqslant 4$

$$
\max \left|t_{i j}^{[k]}\right| \leqslant \begin{cases}M K^{k} \frac{\left(x+y-x_{0}-y_{0}\right)^{k}}{k !}, & \text { если } i+j=0,1, \\ M K^{k} \frac{\left(x+y-x_{0}-y_{0}\right)^{k-1}}{(k-1) !}, & \text { если } i+j=2, \\ M K^{k} \frac{\left(x+y-x_{0}-y_{0}\right)^{k-2}}{(k-2) !}, & \text { если } i+j=3, \\ M K^{k} \frac{\left(x+y-x_{0}-y_{0}\right)^{k-3}}{(k-3) !}, & \text { если } i+j=4 .\end{cases}
$$

Из этих оценок видно, что существует $k_{0}<\infty$ такое, что отображение $\mathscr{A}^{k_{0}}$ является сжимаюшим. Это доказывает существование и единственность неподвижной точки для отображения $\mathscr{A}$ и естественньм образом завершает доказательство леммы в случае $n=1$. Ясно, что аналогично устанавливается справедливость леммы и для $n=2,3,4, \ldots$.

СледСтвиЕ 1. Если в уравнении (1) коэффициенты $a, b \in C^{n+1, n+1}(P), c \in$ $C^{n, n}(P), n \geqslant 0$, то его функиия Римана $R\left(x, y ; x_{*}, y_{*}\right)$ принадлежит $C^{n+1, n+1}(P)$ по каждой паре переменных $(x, y),\left(x_{*}, y_{*}\right)$.

2. О корректности задачи Гурса в классе $W_{2}^{1}$. Известно, что если функция $u(x, y) \in W_{2}^{1}(P)$ является обобщенным решением уравнения (1) в прямоугольнике $P$, то почти всюду в $P$ она совпадает с функцией $u_{c}(x, y) \in C(\bar{P})$ (как и в работе [1], мы будем назьвать ее непрерывным представителем такого решения). При этом для всех $x^{\prime} \in\left[x_{0}, x_{1}\right]$ функция $u_{c}\left(x^{\prime}, y\right) \in W_{2}^{1}\left(y_{0}, y_{1}\right)$ и для всех $y^{\prime} \in\left[y_{0}, y_{1}\right]$ функция $u_{c}\left(x, y^{\prime}\right) \in W_{2}^{1}\left(x_{0}, x_{1}\right)$. 
Teopema 2. Пусть $a, b \in C^{n+1, n+1}(P), c \in C^{n, n}(P), f(x, y) \in W_{2}^{n, n}(P), \varphi_{1} \in$ $W_{2}^{n+1}\left(x_{0}, x_{1}\right), \varphi_{2} \in W_{2}^{n+1}\left(y_{0}, y_{1}\right), n \geqslant 0, \varphi_{1}\left(x_{0}\right)=\varphi_{2}\left(y_{0}\right)$. B пространстве $W_{2}^{1}(P)$ существует и единственно обобщенное решение $и(x, y)$ уравнения (1), удовлетворяющее условиям (4). Это решение принадлежст пространству $W_{2}^{n+1, n+1}(P), u$ справедлива оченка

$$
\|u\|_{W_{2}^{n+1, n+1}(P)}^{2} \leqslant C_{n G}\left(\|f\|_{W_{2}^{n, n}(P)}^{2}+\left\|\varphi_{1}\right\|_{W_{2}^{n+1}\left(x_{0}, x_{1}\right)}^{2}+\left\|\varphi_{2}\right\|_{W_{2}^{n+1}\left(y_{0}, y_{1}\right)}^{2}\right),
$$

әде $C_{n G}$ не зависит от $f, \varphi_{1}, \varphi_{2}$.

ДокАЗАТЕльство. Все утверждения теоремы следуют из того, что для всякого обобщенного решения $u(x, y) \in W_{2}^{1}(P)$ уравнения (1) справедливо представление (см. [1])

$$
\begin{aligned}
u_{c}\left(x_{*}, y_{*}\right)= & \frac{1}{2} u_{c}\left(x_{*}, y_{0}\right) R\left(x_{*}, y_{0} ; x_{*}, y_{*}\right)+\frac{1}{2} u_{c}\left(x_{0}, y_{*}\right) R\left(x_{0}, y_{*} ; x_{*}, y_{*}\right) \\
& +\int_{y_{0}}^{y_{*}}\left(R\left(x_{0}, y ; x_{*}, y_{*}\right)\left(\frac{1}{2} u_{c}\left(x_{0}, y\right)_{y}^{\prime}+a\left(x_{0}, y\right) u_{c}\left(x_{0}, y\right)\right)\right. \\
& \left.\quad-\frac{1}{2} \frac{\partial R}{\partial y}\left(x_{0}, y ; x_{*}, y_{*}\right) u_{c}\left(x_{0}, y\right)\right) d y \\
& +\int_{x_{0}}^{x_{*}}\left(R\left(x, y_{0} ; x_{*}, y_{*}\right)\left(\frac{1}{2} u_{c}\left(x, y_{0}\right)_{x}^{\prime} 2+b\left(x, y_{0}\right) u_{c}\left(x, y_{0}\right)\right)\right. \\
& \left.-\frac{1}{2} \frac{\partial R}{\partial x}\left(x, y_{0} ; x_{*}, y_{*}\right) u_{c}\left(x, y_{0}\right)\right) d x \\
+ & \int_{x_{0}}^{x_{*}} d x \int_{y_{0}}^{y_{*}} f(x, y) R\left(x, y ; x_{*}, y_{*}\right) d y
\end{aligned}
$$

где $R\left(x, y ; x_{*}, y_{*}\right)$ - функция Римана уравнения (1). Ясно, что искомое решение задачи Гурса получается из этого выражения заменой $u_{c}\left(x_{*}, y_{0}\right)$ на $\varphi_{1}\left(x_{*}\right), u_{c}\left(x_{0}, y_{*}\right)$ на $\varphi_{2}\left(y_{*}\right)$.

3. О корректности задачи Дарбу в классе $W_{2}^{1}$. Пусть теперь уравнение (1) задано в области $S_{D}:=\left\{(x, y): x_{*}<x<0,0<y<l(x)\right\}$, где $l(x) \in C^{n+1}\left[x_{*}, 0\right]$, $l^{\prime}(x)<0$ для всех $x \in\left[x_{*}, 0\right], l(0)=0$, и пусть $a(x, y), b(x, y) \in C^{n+1, n+1}\left(S_{0}\right), c(x, y) \in$ $C^{n, n}\left(S_{0}\right)$, где $S_{0} \supset \bar{S}_{D}$.

ТЕорема 3. Для любой функиии $f \in W_{2}^{n, n}\left(S_{D}\right), n \geqslant 0$, любъх функиий $\varphi_{1}, \varphi_{2} \in$ $W_{2}^{n+1}\left(x_{*}, 0\right), \varphi_{1}(0)=\varphi_{2}(0)$, в пространстве $W_{2}^{1}\left(S_{D}\right)$ существует и единственно обобщенное решение $и(x, y)$ уравнения (1), удовлетворяющее условиям

$$
u(x, 0)=\varphi_{1}(x), \quad u(x, l(x))=\varphi_{2}(x), \quad x \in\left(x_{*}, 0\right)
$$

$Э$ Эо решение принадлежит пространству $W_{2}^{n+1, n+1}\left(S_{D}\right)$, и справедлива оченка

$$
\|u\|_{W_{2}^{n+1, n+1}\left(S_{D}\right)}^{2} \leqslant C_{n D}\left(\|f\|_{W_{2}^{n, n}\left(S_{D}\right)}^{2}+\left\|\varphi_{1}\right\|_{W_{2}^{n+1}\left(x_{*}, 0\right)}^{2}+\left\|\varphi_{2}\right\|_{W_{2}^{n+1}\left(x_{*}, 0\right)}^{2}\right)
$$

әде $C_{n D}$ не зависит от $f, \varphi_{1}, \varphi_{2}$. 
ДокАЗАтЕльство. Легко доказать, что для непрерьвного представителя $u_{c}(x, y)$ всякого решения сформулированной задачи Дарбу при любом $x_{0} \in\left(x_{*}, 0\right)$ справедливо равенство

$$
\begin{aligned}
\varphi_{1}\left(x_{0}\right)= & \frac{1}{2} \varphi_{2}(0) R\left(0,0 ; x_{0}, 0\right)+\frac{1}{2} \varphi_{2}\left(x_{0}\right) R\left(x_{0}, l\left(x_{0}\right) ; x_{0}, 0\right) \\
& +\int_{x_{0}}^{0} \varphi_{2}(x)\left(\frac{1}{2} \frac{\partial R}{\partial x}\left(x, l(x) ; x_{0}, 0\right)-\frac{l^{\prime}(x)}{2} \frac{\partial R}{\partial y}\left(x, l(x) ; x_{0}, 0\right)\right. \\
& \left.\quad-\left(b(x, l(x))-l^{\prime}(x) a(x, l(x))\right) R\left(x, l(x) x ; x_{0}, 0\right)\right) d x \\
& +\int_{x_{0}}^{0} R\left(x, l(x) ; x_{0}, 0\right)\left(\frac{\partial u_{c}}{\partial x}-\left.l^{\prime}(x) \frac{\partial u_{c}}{\partial y}\right|_{y=l(x)}\right) d x \\
& +\int_{x_{0}}^{0} d x \int_{0}^{l(x)} R\left(x, y ; x_{0}, 0\right) f(x, y) d y,
\end{aligned}
$$

где $R=R\left(x, y ; x_{0}, y_{0}\right)$ - функция Римана уравнения (1). Это равенство можно записать в виде

$$
\int_{x_{0}}^{0} R\left(x, l(x) ; x_{0}, 0\right) h_{D}(x) d x=\varphi_{3 D}\left(x_{0}\right) \quad \text { для всех } x_{0} \in\left(x_{*}, 0\right),
$$

где

$$
h_{D}(x):=\frac{\partial u}{\partial x}-\left.l^{\prime}(x) \frac{\partial u}{\partial y}\right|_{y=l(x)}, \quad \varphi_{3 D}(x) \in W_{2}^{1}\left(x_{*}, 0\right) .
$$

Обозначим через $L_{2, *}\left(x_{*}, 0\right)$ гильбертово пространство классов измеримых на этом интервале функций с нормой

$$
\|h(x)\|_{L_{2, *}\left(x_{*}, 0\right)}^{2}=\int_{x_{*}}^{0}|h(x)|^{2}|x| d x .
$$

Из результатов работы [1] следует, что функция $h_{D}(x) \in L_{2, *}\left(x_{*}, 0\right)$. Из (5) для почти всех $x_{0} \in\left(x_{*}, 0\right)$ получаем равенство

$$
-R\left(x_{0}, l\left(x_{0}\right) ; x_{0}, 0\right) h_{D}\left(x_{0}\right)+\int_{x_{0}}^{0}\left(R\left(x, l(x) ; x_{0}, 0\right)\right)_{x_{0}}^{\prime} h_{D}(x) d x=\varphi_{3 D}^{\prime}\left(x_{0}\right) .
$$

Поскольку функция $R\left(x, l(x) ; x_{0}, 0\right)$ непрерывна вместе со своими первыми частными производньги по переменным $x, x_{0}$ на множестве $T:=\left\{\left(x_{0}, x\right): x_{*} \leqslant x_{0} \leqslant 0, x_{0} \leqslant x \leqslant 0\right\}$ и $R\left(x_{0}, l\left(x_{0}\right) ; x_{0}, 0\right) \neq 0$, из последнего равенства следует, что на самом деле $h_{D}(x) \in$ $L_{2}\left(x_{0}, x_{1}\right)$. Как известно, для любой функции $\varphi_{4 D}\left(x_{0}\right):=\varphi_{3 D}^{\prime}\left(x_{0}\right) \in L_{2}\left(x_{0}, x_{1}\right)$ в этом пространстве существует единственное решение $h_{D}(x)$ уравнения Вольтерра (6), причем

$$
\left\|h_{D}\right\|_{W_{2}^{n}\left(x_{*}, 0\right)} \leqslant C_{n V}\left\|\varphi_{3 D}\right\|_{W_{2}^{(n+1)}\left(x_{*}, 0\right)}, \quad n=0,1, \ldots,
$$

где $C_{n V}$ не зависит от $\varphi_{3 D}$. Для завершения доказательства теоремы остается лишь заметить, что искомое решение $u(x, y)$ восстанавливается однозначно по функциям $\varphi_{2}, h_{D}$ и $f$ с помощью известной формулы Римана (см., например, [9, с. 263]), справедливость которой для непрерьвных представителей обобщенных решений уравнения (1), принадлежащих $W_{2}^{1}\left(S_{D}\right)$, следует из [1]. 
4. Доказательство теоремы 1. Пусть $u(x, y) \in W_{2}^{1}(D)$ является обобщенным решением задачи $(1),(2)$. Используя результаты работы $[1]$, несложно убедиться в справедливости равенства

$$
\begin{aligned}
& \varphi_{2}\left(x_{0}\right)=\int_{x_{0}}^{\psi_{1}\left(l_{2}\left(x_{0}\right)\right)} d x \int_{l_{2}\left(x_{0}\right)}^{l_{1}(x)} R\left(x, y ; x_{0}, l_{2}\left(x_{0}\right)\right) f(x, y) d y \\
& +\frac{1}{2} \varphi_{1}\left(\psi_{1}\left(l_{2}\left(x_{0}\right)\right)\right) R\left(\psi_{1}\left(l_{2}\left(x_{0}\right)\right), l_{2}\left(x_{0}\right) ; x_{0}, l_{2}\left(x_{0}\right)\right)+\frac{1}{2} \varphi_{1}\left(x_{0}\right) R\left(x_{0}, l_{1}\left(x_{0}\right) ; x_{0}, l_{2}\left(x_{0}\right)\right) \\
& +\int_{x_{0}}^{\psi_{1}\left(l_{2}\left(x_{0}\right)\right)} \varphi_{1}(x)\left(\frac{1}{2} \frac{\partial R}{\partial x}\left(x, l_{1}(x) ; x_{0}, l_{2}\left(x_{0}\right)\right)-\frac{l_{1}^{\prime}(x)}{2} \frac{\partial R}{\partial y}\left(x, l_{1}(x) ; x_{0}, l_{2}\left(x_{0}\right)\right)\right. \\
& \left.\quad-\left(b\left(x, l_{1}(x)\right)-l_{1}^{\prime}(x) a\left(x, l_{1}(x)\right)\right) R\left(x, l_{1}(x) ; x_{0}, l_{2}\left(x_{0}\right)\right)\right) d x \\
& +\int_{x_{0}}^{\psi_{1}\left(l_{2}\left(x_{0}\right)\right)} R\left(x, l_{1}(x) ; x_{0}, l_{2}\left(x_{0}\right)\right)\left(\frac{\partial u}{\partial x}-\left.l_{1}^{\prime}(x) \frac{\partial u}{\partial y}\right|_{y=l_{1}(x)}\right) d x
\end{aligned}
$$

где $R\left(x, y ; x_{0}, y_{0}\right)$ - функция Римана уравнения $(1)$, а $\psi_{1}(x)$-обратная к $y=l_{1}(x)$ функция. Положим

$$
h(x):=\frac{\partial u}{\partial x}-\left.l_{1}^{\prime}(x) \frac{\partial u}{\partial y}\right|_{y=l_{1}(x)} .
$$

Из работы [1] следует, что $h(x) \in L_{2, *}\left(x_{*}, 0\right)$. Для любой точки $x \in\left[x_{*}, 0\right)$ определим отображение $\Lambda: x \mapsto \psi_{1}\left(l_{2}(x)\right), \Lambda(0)=0$ (некоторые его свойства описаны в леммах 2.3-2.5 из [1]). Тогда равенство (7) можно записать в виде

$$
\int_{\Lambda\left(x_{0}\right)}^{x_{0}} R\left(x, l_{1}(x) ; x_{0}, l_{2}\left(x_{0}\right)\right) h(x) d x=\varphi_{3}\left(x_{0}\right) \quad \text { для всех } \quad x_{0} \in\left(x_{*}, 0\right),
$$

где $\varphi_{3}(x) \in W_{2}^{1}\left(x_{*}, 0\right)$. Дифференцируя последнее равенство и используя свойства функции Римана, получаем

$$
h\left(x_{0}\right)=\Lambda^{\prime}\left(x_{0}\right) E\left(x_{0}\right) h\left(\Lambda\left(x_{0}\right)\right)+\int_{\Lambda\left(x_{0}\right)}^{x_{0}} K\left(x, x_{0}\right) h(x) d x+\varphi_{4}\left(x_{0}\right)
$$

для почти всех $x_{0} \in\left(x_{*}, 0\right)$, в котором

$$
\begin{gathered}
E\left(x_{0}\right):=\exp \left(\int_{\Lambda\left(x_{0}\right)}^{x_{0}} b\left(x, l_{2}\left(x_{0}\right)\right) d x-\int_{l_{2}\left(x_{0}\right)}^{l_{1}\left(x_{0}\right)} a\left(x_{0}, y\right) d y\right), \\
K\left(x, x_{0}\right):=\left(R\left(x, l_{1}(x) ; x_{0}, l_{2}\left(x_{0}\right)\right)\right)_{x_{0}}^{\prime} \exp \left(-\int_{l_{2}\left(x_{0}\right)}^{l_{1}\left(x_{0}\right)} a\left(x_{0}, y\right) d y\right),
\end{gathered}
$$

а $\varphi_{4} \in L_{2}\left(x_{*}, 0\right)$. Рассмотрим цепочку равенств, эквивалентных (8):

$$
\begin{aligned}
& \prod_{j=0}^{i-1} \Lambda^{\prime}\left(\Lambda^{j}\left(x_{0}\right)\right) \prod_{j=0}^{i-1} E\left(\Lambda^{j}\left(x_{0}\right)\right) h\left(\Lambda^{i}\left(x_{0}\right)\right)=\prod_{j=0}^{i} \Lambda^{\prime}\left(\Lambda^{j}\left(x_{0}\right)\right) \prod_{j=0}^{i} E\left(\Lambda^{j}\left(x_{0}\right)\right) h\left(\Lambda^{i+1}\left(x_{0}\right)\right) \\
& \quad+\int_{\Lambda^{i+1}\left(x_{0}\right)}^{\Lambda^{i}\left(x_{0}\right)} \prod_{j=0}^{i-1} \Lambda^{\prime}\left(\Lambda^{j}\left(x_{0}\right)\right) \prod_{j=0}^{i-1} E\left(\Lambda^{j}\left(x_{0}\right)\right) K\left(x, \Lambda^{i}\left(x_{0}\right)\right) h(x) d x \\
& \quad+\prod_{j=0}^{i-1} \Lambda^{\prime}\left(\Lambda^{j}\left(x_{0}\right)\right) \prod_{j=0}^{i-1} E\left(\Lambda^{j}\left(x_{0}\right)\right) \varphi_{4}\left(\Lambda^{i}\left(x_{0}\right)\right) \quad \text { для почти всех } x_{0} \in\left(x_{*}, 0\right), \quad i=1,2, \ldots
\end{aligned}
$$


Несложно убедиться в том, что последовательность чисел

$$
M_{i}:=\max _{x_{0} \in\left[x_{*}, 0\right]}\left|\prod_{j=0}^{i-1} \Lambda^{\prime}\left(\Lambda^{j}\left(x_{0}\right)\right)\right| \rightarrow 0, \quad i \rightarrow \infty
$$

мажорируется сходящейся геометрической прогрессией, а последовательность непрерывных функций

$$
e_{i}\left(x_{0}\right):=\prod_{j=0}^{i-1} E\left(\Lambda^{j}\left(x_{0}\right)\right)
$$

равномерно ограничена на отрезке $\left[x_{*}, 0\right]$. Поскольку

$$
\begin{aligned}
& \left\|\varphi_{4}\left(\Lambda^{i}\left(x_{0}\right)\right) \prod_{j=0}^{i-1} \Lambda^{\prime}\left(\Lambda^{j}\left(x_{0}\right)\right)\right\|_{L_{2}}^{2}=\int_{x_{*}}^{0}\left|\prod_{j=0}^{i-1} \Lambda^{\prime}\left(\Lambda^{j}\left(x_{0}\right)\right) \varphi_{4}\left(\Lambda^{i}\left(x_{0}\right)\right)\right|^{2} d x_{0} \\
& \leqslant M_{i} \int_{x_{*}}^{0}\left|\prod_{j=0}^{i-1} \Lambda^{\prime}\left(\Lambda^{j}\left(x_{0}\right)\right)\right| \cdot\left|\varphi_{4}\left(\Lambda^{i}\left(x_{0}\right)\right)\right|^{2} d x_{0}=M_{i} \int_{\Lambda\left(x_{*}\right)}^{0}\left|\varphi_{4}(z)\right|^{2} d z
\end{aligned}
$$

ряд

$$
\sum_{i=1}^{\infty}\left(\prod_{j=0}^{i-1} \Lambda^{\prime}\left(\Lambda^{j}\left(x_{0}\right)\right) \prod_{j=0}^{i-1} E\left(\Lambda^{j}\left(x_{0}\right)\right) \varphi_{4}\left(\Lambda^{i}\left(x_{0}\right)\right)\right)
$$

сходится в пространстве $L_{2}\left(x_{*}, 0\right)$. А так как

$$
\begin{aligned}
& \int_{x_{*}}^{0}\left|\int_{\Lambda^{i+1}\left(x_{0}\right)}^{\Lambda^{i}\left(x_{0}\right)} \prod_{j=0}^{i-1} \Lambda^{\prime}\left(\Lambda^{j}\left(x_{0}\right)\right) \prod_{j=0}^{i-1} E\left(\Lambda^{j}\left(x_{0}\right)\right) K\left(x, \Lambda^{i}\left(x_{0}\right)\right) h(x) d x\right|^{2} d x_{0} \\
& \quad \leqslant C M_{i}^{2} \int_{x_{*}}^{0}\left|\Lambda^{i+1}\left(x_{0}\right)-\Lambda^{i}\left(x_{0}\right)\right|\left(\int_{\Lambda^{i+1}\left(x_{0}\right)}^{\Lambda^{i}\left(x_{0}\right)}|h(x)|^{2} d x\right) d x_{0} \\
& \quad \leqslant C M_{i}^{2} \int_{x_{*}}^{0} \frac{\left|\Lambda^{i+1}\left(x_{0}\right)-\Lambda^{i}\left(x_{0}\right)\right|}{\left|\Lambda^{i+1}\left(x_{0}\right)\right|}\left(\int_{\Lambda^{i+1}\left(x_{0}\right)}^{\Lambda^{i}\left(x_{0}\right)}|h(x)|^{2}|x| d x\right) d x_{0} \\
& \quad \leqslant C_{1} M_{i}^{2} \int_{x_{*}}^{0}|h(x)|^{2}|x| d x
\end{aligned}
$$

ряд

$$
\sum_{i=1}^{\infty}\left(\int_{\Lambda^{i+1}\left(x_{0}\right)}^{\Lambda^{i}\left(x_{0}\right)} \prod_{j=0}^{i-1} \Lambda^{\prime}\left(\Lambda^{j}\left(x_{0}\right)\right) \prod_{j=0}^{i-1} E\left(\Lambda^{j}\left(x_{0}\right)\right) K\left(x, \Lambda^{i}\left(x_{0}\right)\right) h(x) d x\right)
$$

также сходится в пространстве $L_{2}\left(x_{*}, 0\right)$. Очевидно, что ряды $(10),(11)$ сходятся и в пространстве $L_{2, *}\left(x_{*}, 0\right)$. Складывая $i$ равенств $(9)$, приходим к вьводу, что последовательность функций

$$
h_{(i)}\left(x_{0}\right):=h\left(x_{0}\right)-\prod_{j=0}^{i} \Lambda^{\prime}\left(\Lambda^{j}\left(x_{0}\right)\right) \prod_{j=0}^{i} E\left(\Lambda^{j}\left(x_{0}\right)\right) h\left(\Lambda^{i+1}\left(x_{0}\right)\right)
$$


является последовательностью Коши в $L_{2, *}\left(x_{*}, 0\right)$. А так как $h(x) \in L_{2, *}\left(x_{*}, 0\right)$, то и $h_{(i)}\left(x_{0}\right)-h\left(x_{0}\right)$ также является последовательностью Коши в этом пространстве, причем

$$
\begin{aligned}
& \left\|\prod_{j=0}^{i} \Lambda^{\prime}\left(\Lambda^{j}\left(x_{0}\right)\right) \prod_{j=0}^{i} E\left(\Lambda^{j}\left(x_{0}\right)\right) h\left(\Lambda^{i+1}\left(x_{0}\right)\right)\right\|_{L_{2, x}\left(x_{*}, 0\right)}^{2} \\
& \quad=\int_{x_{*}}^{0}\left|\prod_{j=0}^{i} \Lambda^{\prime}\left(\Lambda^{j}\left(x_{0}\right)\right) \prod_{j=0}^{i} E\left(\Lambda^{j}\left(x_{0}\right)\right) h\left(\Lambda^{i+1}\left(x_{0}\right)\right)\right|^{2}\left|x_{0}\right| d x_{0} \\
& \quad \leqslant C \int_{x_{*}}^{0}\left|\prod_{j=0}^{i} \Lambda^{\prime}\left(\Lambda^{j}\left(x_{0}\right)\right) \frac{\left|x_{0}\right|}{\Lambda^{i+1}\left(x_{0}\right)}\right| \cdot\left|h^{2}\left(\Lambda^{i+1}\left(x_{0}\right)\right) \Lambda^{i+1}\left(x_{0}\right)\left(\Lambda^{i+1}\left(x_{0}\right)\right)^{\prime}\right| d x_{0} .
\end{aligned}
$$

Положим

$$
q:=\lim _{x \rightarrow-0} \frac{l_{2}(x)}{l_{1}(x)} .
$$

Из условия (3) следует, что $0<q<1$. Фиксируем $\varepsilon>0$ такое, что

$$
q_{1}:=\frac{(q+\varepsilon)^{2}}{q-\varepsilon}<1 .
$$

Тогда существует $x_{1} \in\left(x_{*}, 0\right)$ такое, что для всех $x_{0} \in\left(x_{1}, 0\right)$ выполнены неравенства

$$
\left|\Lambda^{\prime}\left(x_{0}\right)\right|>q-\varepsilon, \quad\left|\Lambda^{i}\left(x_{0}\right)\right|<(q+\varepsilon)^{i}\left|x_{0}\right|, \quad i=1,2, \ldots .
$$

Несложно убедиться в справедливости равенства

$$
\begin{aligned}
& \Lambda^{i+1}\left(x_{0}\right)=\Lambda^{\prime}\left(\Lambda^{i}\left(x_{0}\right)\right) \Lambda^{\prime}\left(\Lambda^{i-1}\left(x_{0}\right)\right) \cdots \Lambda^{\prime}\left(\Lambda\left(x_{0}\right)\right) \Lambda^{\prime}\left(x_{0}\right) x_{0} \\
& \quad-\frac{\Lambda^{\prime \prime}\left(\xi_{1}\right)}{2} x_{0}^{2} \Lambda^{\prime}\left(\Lambda\left(x_{0}\right)\right) \Lambda^{\prime}\left(\Lambda^{2}\left(x_{0}\right)\right) \cdots \Lambda^{\prime}\left(\Lambda^{i}\left(x_{0}\right)\right) \\
& \quad-\frac{\Lambda^{\prime \prime}\left(\xi_{2}\right)}{2}\left(\Lambda\left(x_{0}\right)\right)^{2} \Lambda^{\prime}\left(\Lambda^{2}\left(x_{0}\right)\right) \Lambda^{\prime}\left(\Lambda^{3}\left(x_{0}\right)\right) \cdots \Lambda^{\prime}\left(\Lambda^{i}\left(x_{0}\right)\right) \\
& \quad-\frac{\Lambda^{\prime \prime}\left(\xi_{3}\right)}{2}\left(\Lambda^{2}\left(x_{0}\right)\right)^{2} \Lambda^{\prime}\left(\Lambda^{3}\left(x_{0}\right)\right) \Lambda^{\prime}\left(\Lambda^{4}\left(x_{0}\right)\right) \cdots \Lambda^{\prime}\left(\Lambda^{i}\left(x_{0}\right)\right)-\cdots-\frac{\Lambda^{\prime \prime}\left(\xi_{i+1}\right)}{2}\left(\Lambda^{i}\left(x_{0}\right)\right)^{2}
\end{aligned}
$$

где $\xi_{i} \in\left[x_{1}, 0\right]$. Поэтому если $K_{2}:=\max _{x \in\left[x_{1}, 0\right]}\left|\Lambda^{\prime \prime}(x)\right|$, то

$$
\begin{aligned}
\left|\prod_{j=0}^{i} \Lambda^{\prime}\left(\Lambda^{j}\left(x_{0}\right)\right) \frac{\left|x_{0}\right|}{\Lambda^{i+1}\left(x_{0}\right)}\right| \leqslant & \left(1-K_{2}\left(\frac{\left|x_{0}\right|}{\left|\Lambda^{\prime}\left(x_{0}\right)\right|}+\frac{\left(\Lambda\left(x_{0}\right)\right)^{2}}{\left|x_{0} \Lambda^{\prime}\left(x_{0}\right) \Lambda^{\prime}\left(\Lambda\left(x_{0}\right)\right)\right|}+\cdots\right.\right. \\
& \left.\left.+\frac{\left(\Lambda^{i}\left(x_{0}\right)\right)^{2}}{\left|x_{0} \Lambda^{\prime}\left(x_{0}\right) \Lambda^{\prime}\left(\Lambda\left(x_{0}\right)\right) \cdots \Lambda^{\prime}\left(\Lambda^{i}\left(x_{0}\right)\right)\right|}\right)\right)^{-1} \\
\leqslant & \left(1-\frac{K_{2}\left|x_{0}\right|}{q-\varepsilon}\left(1+\frac{(q+\varepsilon)^{2}}{q-\varepsilon}+\cdots+\frac{(q+\varepsilon)^{2 i}}{(q-\varepsilon)^{i}}\right)\right)^{-1} \\
\leqslant & \left(1-\frac{K_{2}\left|x_{0}\right|}{(q-\varepsilon)-(q+\varepsilon)^{2}}\right)^{-1} .
\end{aligned}
$$


Отсюда видно, что существуют значение $x_{2} \in\left(x_{1}, 0\right)$ и положительная постоянная $C_{3}$, не зависящая от номера $i$, такие, что

$$
\left|\prod_{j=0}^{i} \Lambda^{\prime}\left(\Lambda^{j}\left(x_{0}\right)\right) \frac{\left|x_{0}\right|}{\Lambda^{i+1}\left(x_{0}\right)}\right| \leqslant C_{3} \quad \text { для всех } x_{0} \in\left(x_{2}, 0\right) .
$$

Поэтому

$$
\begin{aligned}
& \left\|\prod_{j=0}^{i} \Lambda^{\prime}\left(\Lambda^{j}\left(x_{0}\right)\right) \prod_{j=0}^{i} E\left(\Lambda^{j}\left(x_{0}\right)\right) h\left(\Lambda^{i+1}\left(x_{0}\right)\right)\right\|_{L_{2, *}\left(x_{2}, 0\right)}^{2} \\
& \quad \leqslant C C_{3} \int_{x_{2}}^{0}\left|h^{2}\left(\Lambda^{i+1}\left(x_{0}\right)\right) \Lambda^{i+1}\left(x_{0}\right)\left(\Lambda^{i+1}\left(x_{0}\right)\right)^{\prime}\right| d x_{0} \\
& \quad \leqslant C C_{3} \int_{\Lambda^{i+1}\left(x_{2}\right)}^{0}|h(z)|^{2}|z| d z \rightarrow 0, \quad i \rightarrow \infty .
\end{aligned}
$$

Отсюда следует, что при почти всех значениях $x_{0} \in\left(x_{2}, 0\right)$ справедливо равенство

$$
\begin{aligned}
h\left(x_{0}\right)= & \int_{\Lambda\left(x_{0}\right)}^{x_{0}} K\left(x, x_{0}\right) h(x) d x+\varphi_{4}\left(x_{0}\right) \\
& +\sum_{i=1}^{\infty}\left(\int_{\Lambda^{i+1}\left(x_{0}\right)}^{\Lambda^{i}\left(x_{0}\right)} \prod_{j=0}^{i-1} \Lambda^{\prime}\left(\Lambda^{j}\left(x_{0}\right)\right) \prod_{j=0}^{i-1} E\left(\Lambda^{j}\left(x_{0}\right)\right) K\left(x, \Lambda^{i}\left(x_{0}\right)\right) h(x) d x\right) \\
& +\sum_{i=1}^{\infty}\left(\prod_{j=0}^{i-1} \Lambda^{\prime}\left(\Lambda^{j}\left(x_{0}\right)\right) \prod_{j=0}^{i-1} E\left(\Lambda^{j}\left(x_{0}\right)\right) \varphi_{4}\left(\Lambda^{i}\left(x_{0}\right)\right)\right),
\end{aligned}
$$

а поскольку ряды справа сходятся в $L_{2}\left(x_{2}, 0\right)$, то, очевидно, $h\left(x_{0}\right) \in L_{2}\left(x_{2}, 0\right)$ и, следовательно, $h\left(x_{0}\right) \in L_{2}\left(x_{*}, 0\right)$. Но тогда

$$
\left\|\prod_{j=0}^{i} \Lambda^{\prime}\left(\Lambda^{j}\left(x_{0}\right)\right) \prod_{j=0}^{i} E\left(\Lambda^{j}\left(x_{0}\right)\right) h\left(\Lambda^{i+1}\left(x_{0}\right)\right)\right\|_{L_{2, x}\left(x_{*}, 0\right)}^{2} \rightarrow 0, \quad i \rightarrow \infty,
$$

поэтому равенство (12) на самом деле справедливо при почти всех $x_{0} \in\left(x_{*}, 0\right)$. Положим

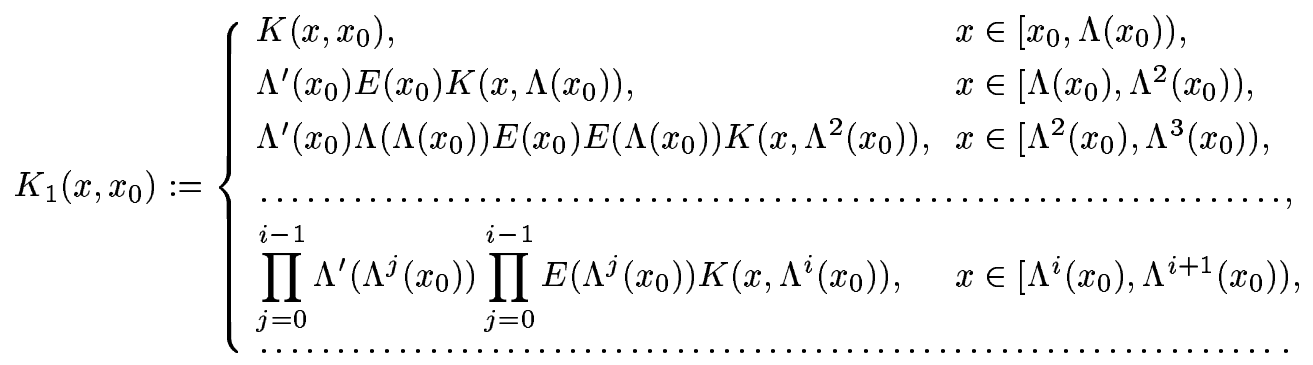

Тогда равенство (12) можно записать в виде

$$
h\left(x_{0}\right)=\int_{x_{0}}^{0} K_{1}\left(x, x_{0}\right) h(x) d x+\varphi_{5}\left(x_{0}\right) \quad \text { для почти всех } x_{0} \in\left(x_{*}, 0\right),
$$


где функция $\varphi_{5}\left(x_{0}\right) \in L_{2}\left(x_{*}, 0\right)$, а функция $K_{1}\left(x, x_{0}\right)$ определена в области $T=\left\{\left(x_{0}, x\right)\right.$ : $\left.x_{*}<x_{0}<0, x_{0}<x<0\right\}$, имеет бесконечное число линий разрьва, задаваемых уравнениями $x=\Lambda^{i}\left(x_{0}\right), i=1,2, \ldots$, но при этом ограничена в этой области, а именно, существует постоянная $k_{1}>0$ такая, что $\left|K_{1}\left(x, x_{0}\right)\right| \leqslant k_{1}$ для всех $\left(x_{0}, x\right) \in T$. Поэтому уравнение (13) в пространстве $L_{2}\left(x_{*}, 0\right)$ обязательно имеет, притом единственное, решение $h\left(x_{0}\right)$, и справедлива оценка

$$
\|h\|_{L_{2}\left(x_{*}, 0\right)}^{2} \leqslant C_{V}\left\|\varphi_{5}\right\|_{L_{2}\left(x_{*}, 0\right)}^{2} \leqslant C_{4}\left(\|f\|_{L_{2}(D)}^{2}+\left\|\varphi_{1}\right\|_{W_{2}^{1}\left(x_{*}, 0\right)}^{2}+\left\|\varphi_{2}\right\|_{W_{2}^{1}\left(x_{*}, 0\right)}^{2}\right) .
$$

Если же вьполнены условия второй части теоремы 1 и $n \geqslant 1$, то $K\left(x, x_{0}\right) \in C^{n, n}\left(T_{1}\right)$, где $T_{1}:=\left\{\left(x_{0}, x\right): x_{*}<x_{0}<0, x_{0}<x<\Lambda\left(x_{0}\right)\right\}, E\left(x_{0}\right) \in C^{n}\left[x_{*}, 0\right], \Lambda\left(x_{0}\right) \in C^{n+1}\left[x_{*}, 0\right]$, a функция $\varphi_{5}\left(x_{0}\right) \in W_{2}^{n}\left(x_{*}, 0\right)$. Поэтому если $\mathscr{A}_{1}: W_{2}^{n}\left(x_{*}, 0\right) \rightarrow W_{2}^{n}\left(x_{*}, 0\right)$ - отображение, определяемое правой частью равенства (13), то, используя стандартные рассуждения, несложно установить, что последовательность функций из $W_{2}^{n}\left(x_{*}, 0\right)$ :

$$
\theta_{0} \equiv 0, \quad \theta_{1}:=\varphi_{5}, \quad \theta_{k+1}:=\mathscr{A}_{1}\left[\theta_{k}\right], \quad k=1,2, \ldots
$$

является последовательностью Коши в этом пространстве, поэтому ее предел - функция $h\left(x_{0}\right)$ - также принадлежит $W_{2}^{n}\left(x_{*}, 0\right)$ и

$$
\|h\|_{W_{2}^{n}\left(x_{*}, 0\right)}^{2} \leqslant C_{V n}\left\|\varphi_{5}\right\|_{W_{2}^{n}\left(x_{*}, 0\right)}^{2}
$$

Поскольку искомое решение $u(x, y)$ нашей задачи может быть теперь однозначно восстановлено во всей области $D$ с помощью формулы Римана по функциям $\varphi_{2}, h$ и $f$, то теорема 1 доказана.

СлЕДСТВИЕ 2. Пусть $\varphi_{i}=0, i=1,2$. На пространстве $L_{2}(D)$ определим оператор L, ставящий в соответствие всякой функции $f(x, y)$ решение задачи (1), $(2)$. Из теорем 1-3 следует, что оператор L вполне непрерывен и его спектр состоит из единственной точки $\lambda=0$, которая не является собственным значением, m.e. $L$ - оператор Вольтерра.

ЗАмЕчаниЕ 1. Если рассматривать задачу (1), (2) как задачу об определении положения участка колеблющейся струны

$$
\frac{\partial^{2} u}{\partial t^{2}}-\frac{\partial^{2} u}{\partial s^{2}}=f,\left.\quad u\right|_{s=c_{i} t}=0, \quad 0<c_{1}<1, \quad-1<c_{2}<0
$$

заключенного между двумя точками, в которых положение струны известно (в начальный момент эти точки совпадают, а с течением времени они расходятся в разные стороны), то для решения $u(s, t)$ такой задачи из теорем $1-3$ легко следует энергетическое неравенство

$$
\int_{c_{2} T}^{c_{1} T}\left(\frac{\partial u}{\partial t}\right)^{2}+\left.\left(\frac{\partial u}{\partial s}\right)^{2}\right|_{t=T} d s \leqslant C(T) \iint_{\Delta}|f|^{2} d s d t
$$

где $\Delta:=\left\{(s, t): 0<t<T, c_{2} t<s<c_{1} t\right\}$.

ЗАмЕчАниЕ 2. В [10] для случая, когда $a=b=c=0$, a $\Gamma_{1}, \Gamma_{2}$ являются прямьми, получены оценки для решения задачи (1), (2) в весовых пространствах Соболева, введенных в [11]. Предложенный выше метод исследования задачи также позволяет рассматривать ее в этих пространствах. 
ЗАмЕчАнИЕ 3. Известно, что проблема существования не почти периодических по времени решений задачи Соболева о малых колебаниях вращающейся жидкости, заполняющей область $G$, тесно связана с вопросом о единственности в пространстве $W_{2}^{1}(G)$ обобщенного решения задачи Дирихле для некоторого гиперболического уравнения, заданного в области $G$, и сильно зависит от конфигурации $G$ (см. [1], [7], [12], [13]). Теоремы 1-3 позволяют легко конструировать области (как двумерные, так и трехмерные), для которых имеет место единственность решения такой задачи Дирихле для гиперболических уравнений. Так, например, пусть уравнение (1) задано в области $G$ плоскости $O x y$, ограниченной кривой

$$
\left(r \cos \left(\varphi+\frac{\pi}{4}\right)+1\right)\left(r \sin \left(\varphi+\frac{\pi}{4}\right)+1\right)=a^{2}, \quad a>1 .
$$

Тогда из теорем 1-3 следует, что если сушествует обобщенное решение $u(x, y) \in W_{2}^{1}(G)$ данного уравнения, то оно единственно в этом пространстве. Некоторые другие примеры двумерных и трехмерных областей, обладающих этим свойством, приведены в [14].

ЗАмЕчАниЕ 4. В отличие от классической задачи Дарбу, условие (3), требующее чтобы кривые $\Gamma_{1}, \Gamma_{2}$ не касались характеристик в точке $x=0$, необходимо для корректности задачи $(1),(2)$. Действительно, пусть уравнение (1), в котором $a=b=c=0$ и $f=0$, задано в области $D_{\mathrm{bad}}$, ограниченной кривыми $y=-x, y=x^{2}, x_{*}=-1 / 2$, и пусть $\varphi_{1}=\varphi_{2} \equiv 0$, т.е. вьполнены все условия теоремы 1 кроме условия (3). Пусть $h(t) \neq 0$ - произвольная непрерьвная на интервале $\left(x_{*}, 0\right)$ функция, удовлетворяющая условию

$$
\int_{x}^{-x^{2}} h(t) d t \equiv 0 \quad \text { для всех } x \in\left(x_{*}, 0\right) .
$$

Тогда задача (1), (2) кроме тривиального имеет также решение

$$
u(x, y)=\int_{-y}^{x} h(t) d t,
$$

в чем легко убедиться непосредственной проверкой. Покажем, например, что $u_{x}^{\prime} \in$ $L_{2}\left(D_{\text {bad }}\right)$. Имеем

$$
\iint_{D_{\text {bad }}}\left|\frac{\partial u}{\partial x}\right|^{2} d x=\int_{x_{*}}^{0}|h(x)|^{2}\left|x+x^{2}\right| d x .
$$

Поскольку $h(x)+2 x h\left(-x^{2}\right) \equiv 0$, то

$$
\begin{aligned}
a_{i} & :=\int_{-\left|x_{*}\right|^{2^{i}}}^{-\left|x_{*}\right|^{i+1}}|h(x)|^{2}|x| d x=\int_{-\left|x_{*}\right|^{2^{i}}}^{-\left|x_{*}\right|^{2^{i+1}}} 4|x|^{3}\left|h\left(-x^{2}\right)\right|^{2} d x \\
& =\int_{-\left|x_{*}\right|^{2}}^{-\left|x_{*}\right|^{2^{i+1}}} 2|h(t)|^{2}|t| d t=2 a_{i+1},
\end{aligned}
$$

откуда следует существование интеграла

$$
\int_{x_{*}}^{0}|h(x)|^{2}|x| d x=\sum_{i=0}^{+\infty} a_{i}<\infty,
$$

а вместе с ним и существование интеграла (14). Таким образом, задача (1), (2) в области $D_{\text {bad }}$ некорректна. 


\section{СПИСОК ЦИТИРОВАННОЙ ЛИТЕРАТУРЫ}

[1] Троицкая С. Д. О не почти периодичности решений задачи С. Л. Соболева в областях с ребрами // Изв. РАН. Сер. матем. 1994. Т. 58. № 4. С. 97-124.

[2] Капустин Н. Ю., Шония З.В. К вопросу об обобщенной разрешимости первой задачи Дарбу // Дифференц. уравнения. 1988. Т. 24. №1. С. 85-91.

[3] Капустин Н. Ю. О двух задачах для гиперболического уравнения в характеристическом треугольнике // Докл. АН СССР. 1987. Т. 293. № 2. С. 301-305.

[4] Кальменов Т. Ш., Садыбеков М. А. О задаче Дирихле и нелокальных краевых задачах для волнового уравнения // Дифференц. уравнения. 1990. Т. 26. №1. С. 60-65.

[5] Коврижкин В. В. О слабых решениях задачи Дарбу // Дифференц. уравнения. 1972. Т. 8. № 1. C. $68-75$.

[6] Врагов В. Н. О задачах Гурса и Дарбу для одного класса гиперболических уравнений // Дифференц. уравнения. 1972. Т. 8. № 1. С. 7-16.

[7] Ralston J. V. On stationary models in inviscid rotating fluids // J. Math. Anal. Appl. 1973. V. 44. № 2. P. 366-383.

[8] Троицкая С. Д. О единственности обобщенного решения задачи Дарбу // УМН. 1996. T. 51. № 5. С. 149-150.

[9] Владимиров В. С. Уравнения математической физики. М.: Наука, 1976.

[10] Харибегашвили С. С. Граничные задачи для систем линейных дифференциальных уравнений второго порядка гиперболического типа. Дисс. ... к. ф.-м. н. Тбилиси, 1986.

[11] Кондратьев В. А. Краевые задачи для эллиптических уравнений в областях с коническими или угловыми точками // Тр. ММО. 1967. Т. 16. С. 209-292.

[12] Соболев С. Л. Об одной новой задаче математической физики // Изв. АН СССР. Сер. матем. 1954. Т. 18. № 1 . С. 3-50.

[13] Александрян Р. А. Спектральные свойства операторов, порожденных системами дифференциальных уравнений типа С. Л. Соболева // Тр. ММО. 1960. Т. 9. С. 455-505.

[14] Троицкая С. Д. Некоторые спектральные свойства задачи о малых колебаниях вращающейся жидкости. Дисс. ... к.ф.-м.н. М.: МГУ, 1992. 